\title{
CONDITIONING OF A VACUUM GAP BY SPARKS AND ION BOMBARDMENT
}

\author{
G.P. BEUKEMA \\ Fysisch Laboratorium, Rijksuniversiteit Utrecht, Nederland
}

Reccived 27 December 1971

\section{Synopsis}

The influence of sparks and ion bombardment on the emitting properties of the electrodes and consequently on the breakdown voltage of a vacuum gap are investigated. The cathode used was either a stainless steel or a copper needle (radius of the tip 0.10 and $0.15 \mathrm{~mm}$, respectively). The anode was a hollow hemisphere (radius $15 \mathrm{~mm}$ ) of stainless steel. It appears that the fieldenhancement factor $\beta$ can be reduced by sparking from about 100 to 10 for the stainless steel cathode and from about 120 to 30 for the copper cathode. By ion bombardment $\beta$ further decreases to 7 and 20 , respectively. When the cathode is conditioned by ion bombardment, there is a deconditioning without breakdown at very high electric fields.

1. Introduction. The condition of the cathode is very important for the insulating properties of a vacuum gap. For plane-parallel electrodes and small gaps $(d<1 \mathrm{~mm})$ the electrical breakdown can either be cathode- or anode-initiated, but in both cases the prebreakdown field-emission current is determined by the properties of the cathode. These properties can be expressed in terms of the work function $\phi$ of the cathode material, the field-enhancement factor $\beta$ and the emitting area $A$ of protrusions on the cathode. They can be altered by admitting either an active gas (change of $\phi$ ) or an inert gas to a pressure of, say, $10^{-4}$ torr (change of $\beta$ and $A$ by ion bombardment) and by sparks (change of $\beta$ and $A$ ).

The electrodes are often conditioned by sparks; these sparks increase the breakdown voltage. The conditioning is a result of blowing up protrusions by their own field-emission current or by a bombardment of the cathode with anode material. When the cathode is conditioned, $\beta$ decreases and the breakdown voltage rises, reaching a more or less stable value after a number of sparks. However, if $\beta$ decreases, the probability of a nanode-initiated breakdown increases ${ }^{1}$ ) for planeparallel electrodes. Below a certain value of $\beta$, the breakdown becomes anodeinitiated and the condition of the cathode is influenced by the anode. The bombardment of the cathode by anode material can create new protrusions ${ }^{2}$ ). This means that conditioning of plane-parallel electrodes by sparks has a limit, which does not necessarily coincide with the lowest possible value of $\beta$. Another method to 
increase the breakdown voltage of a vacuum gap is to bombard the cathode with heavy particles. Some authors ${ }^{3.4}$ ) have tried to condition the cathode by a gas discharge, but they did not find a significant decreasc of $\beta$. Alpert and Tomaschkc ${ }^{5}$ ) used a pressure rise, which at a given voltage $V$ was smaller than the pressure derived from the Paschen curve at the same value of $V$. Then, the conditions for the ignition of a gas discharge are not fulfilled. For tungsten, a decrease of $\beta$ by a factor of 5 was found, but the authors did not report the consequences for the breakdown mechanism and what happens at voltages just below the onset of a spark.

Recent measurements of Janssen and Jones ${ }^{\circ}$ ) show that the tip radius of a field emitter decreases by ion bombardment; at a fixed voltage they find an enhanced emission, because the local field at the tip is proportional to $1 / r$ and the emitting area to $r^{2}$. Hence, the preexponential term of the Fowler-Nordheim equation is not altered, but the exponential term increases. It should be noted that the purpose of Alpert's investigation was to lower $\beta$ and that of Janssen and Jones to lower the radius of the field-emitter tip, i.e., to enlarge $\beta$ by ion bombardment.

To investigate the conditioning of a stainless steel and a copper cathode by sparks and ion bombardment without the influence of anode material, we used a small cathode, $r i z$. a needle with a tip radius of about $0.1 \mathrm{~mm}$. This surface is still large compared to a protrusion. The tip is fixed in the centre of a hollow hemispherical anode.

2. Theory. 2.1. The Fowler-Nordheim equation. According to one of the theories for electrical breakdown in vacuum, breakdown caused by fieldemission electrons occurs either by thermal instabilities on the cathode (cathodeinitiated breakdown) or on the anode (anode-initiated breakdown). These electrons are assumed to be emitted from protrusions on the surface of the cathode and to obey Fowler-Nordheim's equation ${ }^{7}$ ):

$$
j=\frac{1.54 \times 10^{-6} E^{2}}{\phi t^{2}(y)} \exp \left(-6.83 \times 10^{9} \frac{\phi^{3 / 2} v(y)}{E}\right) \quad\left(\text { in } \mathrm{A} / \mathrm{m}^{2}\right) .
$$

where $j$ is the current density in $\mathrm{A} / \mathrm{m}^{2}, E$ is the electric field in $\mathrm{V} / \mathrm{m}$ at the emitting site, $\phi$ is the work function of this site in $\mathrm{eV} . t(y)$ and $t(y)$ are elliptic functions of the parameter $y^{8}$ ), which is a function of the electric field and the work function:

$$
y=\left(3.79 \times 10^{-5} E^{\frac{1}{2}}\right) / \phi .
$$

If the emitting site consists of $n$ protrusions on the surface of the cathode with work functions $\phi_{i}$, field-enhancement factors $\beta_{i}$ and emitting areas $A_{i}(i=1 \ldots \ldots n)$ respectively, the total current $I$ is given by the relation:

$$
I=1.54 \times 10^{-6} \sum_{i=1}^{n} \frac{\beta_{i}^{2} E_{i}^{2} A_{i}}{\phi_{i} t^{2}\left(y_{i}\right)} \exp \left[-\left(6.83 \times 10^{9} \frac{\phi_{i}^{3 / 2} v\left(y_{i}\right)}{\beta_{i} E_{0}}\right)\right] \text { (in A). }
$$

where $E_{0}$ is the macroscopic field, i.e., for plane-parallel electrodes $E_{0}=V / d$. 
If one protrusion is responsible for the current, eq. (3) gives for an electric field $E=\beta E_{0}$ :

$$
\log \frac{I}{E_{0}^{2}}=\log \left(\frac{1.54 \times 10^{-6} \beta^{2} A}{\phi t^{2}(y)}\right)-2.95 \times 10^{9} \frac{\phi^{3 / 2} v(y)}{\beta} \frac{1}{E_{0}} .
$$

A graph of $\log I / E_{0}^{2}$ versus $1 / E_{0}$ (Fowler-Nordheim plot) yields a straight line with slope $m$ :

$$
m=\frac{\mathrm{d} \log \left(I / E_{0}^{2}\right)}{\mathrm{d}\left(1 / E_{0}\right)}=-2.95 \times 10^{9} \frac{\phi^{3 / 2} s(y)}{\beta},
$$

with $s(y)=v(y)-\frac{1}{2} y[\mathrm{~d} v(y) / \mathrm{d} y] s(y)$ is a slowly varying function of $\left.y^{8}\right)$. In the field range used for d.c. field-emission experiments up to $10^{-6} \mathrm{~A}, s(y)$ varies only a few percent and is about 0.95 . We have taken $s(y)=0.95$ and $t(y)=1$ over the whole field range. The field-enhancement factor $\beta$ can be calculated from (5).

For the calculation of the emitting area $A$ from a Fowler-Nordheim plot, one must take into account that $v(y)$ is a function of $E$ and $\phi$. In the field range used for d.c. field-emission experiments up till $10^{-6} \mathrm{~A}, v(y)$ varies between 0.73 and 0.54 (see fig. 1).

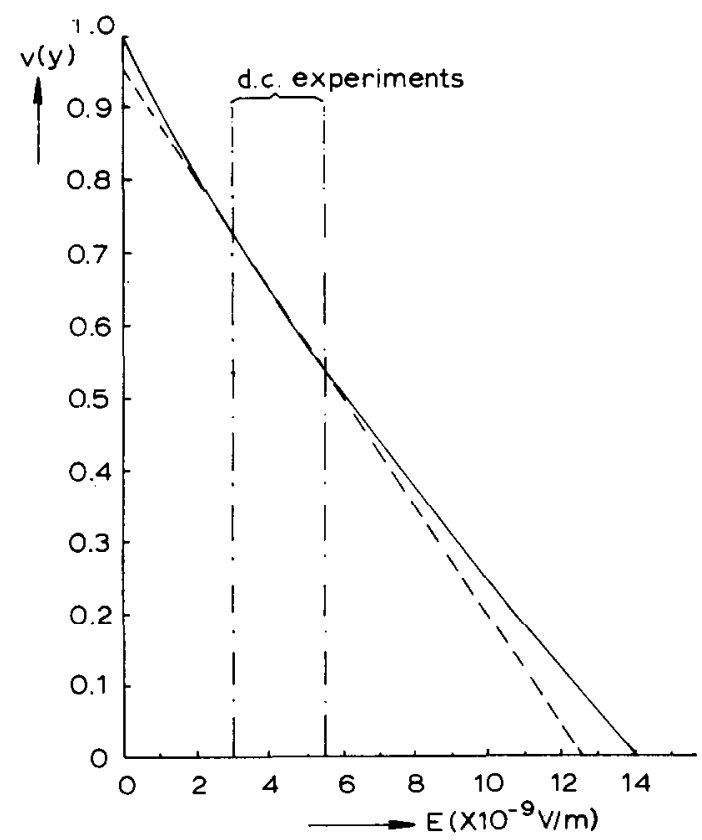

Fig. 1. Full curve: $v(y)$ as a function of the electric field $E$ for $\phi=4.5 \mathrm{eV}$. Broken curve: the approximation $v(y)=0.956-1.062 y^{2}$. 
For current densities between $10^{5} \mathrm{~A} / \mathrm{m}^{2}$ and $10^{8} \mathrm{~A} / \mathrm{m}^{2}$ the following approximation for $v(y)$ is sufficiently accurate:

$$
t(y)=0.956-1.062 y^{2},
$$

and instead of (4) we can write

$$
\log \frac{I}{E_{0}^{2}}=\log 1.54 \times 10^{-6} \frac{\beta^{2} A}{\phi} 10^{4.56 / \sqrt{ } \phi}-2.81 \times 10^{9} \frac{\phi^{3 / 2}}{\beta} \frac{1}{E_{0}} .
$$

If eq. (6) is used instead of $r(y)=1$, as has often been done ${ }^{9,10}$ ), a reduction in the calculated emitting area of about a factor 100 is found.

Another method to calculate $A$ from a Fowler-Nordheim plot is to use Dolan's tables $\left.{ }^{11}\right)$. But this method is unsuitable to calculate $\beta$ and $A$ with help of a simple computer programme.

2.2. Breakdown initiation by field-emission electrons. For planeparallel electrodes we must take into account heating of the anode by the fieldemission electrons. The total power input, $I V$, due to the field-emission current is dissipated by the anode. Calculations of the critical power input ${ }^{9,12}$ ) are based on a uniform disk-heating model with infinite heat sink. But it is questionable whether the disk-heating model may be applied. If $c(y)$ is taken according to eq. (6) the calculated emitting area is about a factor 100 smaller than for $t(y)=1$. Then, the radius of the bombarded area can be of the same order of magnitude as the depth of penetration of the electrons arriving at the anode ${ }^{13}$ ).

Because it is sometimes difficult to derive the breakdown mechanism from the experimental data and to avoid any influence of the anode material on the emitting properties of the cathode, we have taken an electrode configuration as shown in fig. 2. Local heating effects at the anode can be neglected in this configuration.

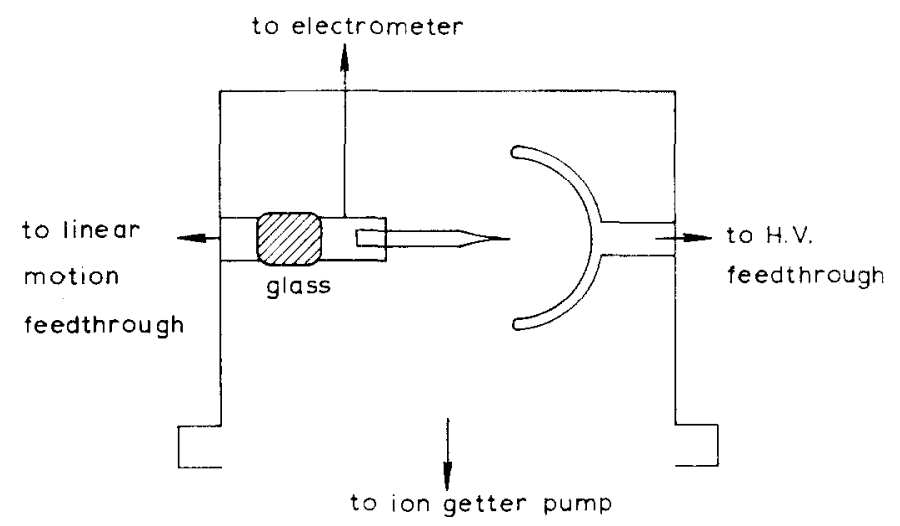

Fig. 2. Electrode configuration; the cathode is a needle of stainless steel or copper with tip radius 0.10 and $0.15 \mathrm{~mm}$, respectively. The stainless steel anode has a radius of $15 \mathrm{~mm}$. 
At the cathode, resistive and Nottingham heating of the protrusions occur due to the field-emission current.

The two protrusion models mostly considered in literature are a truncated cone and a cylinder both topped by a hemisphere. For the field-enhancement factor the following expressions have been derived $\left.{ }^{12,14}\right) . \beta=h / 2 r_{\mathrm{c}}+5$ for a truncated cone with half angle $10^{\circ}$ and $\beta=h / r_{\mathrm{c}}+2$ for a cylinder, where $h$ is the height of the protrusion and $r_{\mathrm{c}}$ is the radius of the tip.

From the investigations by Vibrans ${ }^{14}$ ) we derived an expression for the critical current $I_{\mathrm{c}}$ at which melting of the cylindrical protrusion occurs by resistive heating alone:

$$
I_{\mathrm{c}}=C\left(\frac{\varkappa}{\varrho_{0}} \pi A\right)^{\frac{1}{2}} \frac{1}{\beta-2} \quad(\text { in } \mathrm{A}) .
$$

For a truncated cone $\left(\theta=10^{\circ}\right)$ the critical current is given by the equation:

$$
I_{\mathrm{c}}=0.17 C\left(\frac{x}{\varrho_{0}} \pi A\right)^{\frac{1}{2}} \quad(\text { in } \mathrm{A}) .
$$

Here, $C$ is a constant which can be obtained from graphs given by Vibrans ${ }^{14}$ ); for copper and stainless stecl $C=14.5$. Further, $\varkappa$ is the thermal conductivity in $\mathrm{Wm}^{-1} \mathrm{~K}^{-1}, \varrho_{0}$ the resistivity in $\Omega \mathrm{m}$ at $20^{\circ} \mathrm{C}, A$ is the emitting area in $\mathrm{m}^{2}$ and $\beta$ is the field-enhancement factor.

The failure of the resistive-heating theory to explain several experimental facts led to further studies of the basic physical situation and to recognition of a second energy-exchange process, the Nottingham effect, which for most materials has an even greater influence on tip temperature than resistive heating ${ }^{15}$ ).

The Nottingham effect corresponds to absorption by the lattice of an average amount of energy equal to the difference between the average energy $\langle u\rangle$ of the emitted electrons and the average energy $\left\langle u^{\prime}\right\rangle$ of the charge carriers which fill the vacancies created by the emitted electrons. In the case of field emission, heating occurs at low temperatures changing to cooling above a transition temperature $T_{\mathrm{c}}$. Taking into account the energy distribution of the emitted electrons, Charbonnier $e t a l .{ }^{15}$ ) have calculated the transition temperature and found:

$$
T_{\mathrm{c}}=5.32 \times 10^{-7} E \phi^{-\frac{1}{2}} \quad(\text { in } \mathrm{K}),
$$

where $E$ is in $\mathrm{V} / \mathrm{m}$ and $\phi$ in $\mathrm{eV}$.

Formula (10) is only true if $\left\langle u^{\prime}\right\rangle$ is equal to the Fermi energy $E_{\mathrm{F}}$. Experimental results by Swanson et al. ${ }^{16}$ ) indicate that the experimental value $T_{\mathrm{c} \text { exp. }}$ is well below $T_{\mathrm{c}}$ according to eq. (10). An anomalously low inversion temperature was obtained both for clean and for zirconium-oxygen-coated tungsten. The observed difference between $\left\langle u^{\prime}\right\rangle$ and $E_{\mathrm{F}}$ is attributed to the fact that conduction processes in tungsten are not well described by the free-electron model as is done for the derivation of $T_{\mathrm{c}}$. 
We do not know whether the conduction processes in copper and in stainless steel can be described by the free-electron model, but we started with the assumption that it is allowed (as is also done in the derivation of the Fowler-Nordheim equation) and we suppose that $\left\langle u^{\prime}\right\rangle=E_{\mathbf{F}}$. When $T_{\mathrm{c}}$ is at least $10 \%$ above the melting temperature $T_{\mathrm{m}}$ of the cathode material, the critical power for melting a protrusion by field-emission (Nottingham) heating can be calculated. At low temperature the amount of energy $q$ given to the lattice for each emitted electron is given by the equation ${ }^{15}$ )

$$
q=1.475 \times 10^{-29} E \phi^{-\frac{1}{2}} \quad \text { (in J). }
$$

For a current density $j$ the power density $Q$ is given by

$$
Q=9.2 \times 10^{-11} j E \phi^{-\frac{1}{2}} \quad\left(\text { in } \mathrm{W} / \mathrm{m}^{2}\right) .
$$

For $E=\beta V / r$ we calculated the critical power $P_{c c}$ at which melting of the protrusion occurs by Nottingham heating.

For a cylinder:

$$
P_{\mathrm{cc}}=\left(5.2 \varkappa T_{\mathrm{m}} r \phi^{\frac{1}{2}} A^{\frac{1}{2}} / \beta^{2}\right) \times 10^{8} \quad(\text { in } \mathrm{W}) ;
$$

for a truncated cone:

$$
P_{\mathrm{cc}}=\left(4.6 x T_{\mathrm{m}} r \phi^{\frac{1}{2}} A^{\frac{1}{2}} / \beta\right) \times 10^{7} \quad(\text { in W }) .
$$

3. Apparatus. The electrode system is schematically shown in fig. 2. The cathode is a needle of stainless steel or copper, with tip radius of $0.10 \mathrm{~mm}$ and $0.15 \mathrm{~mm}$, respectively. The anode is made of stainless steel. The cathode can be moved axially and is adjusted in such a way that the centre of the cathode tip and the centre of the anode coincide. The anode is polished to a mirror finish; the cathode is polished in a similar way.

The stainless-steel vacuum chamber is connected to a $50 \mathrm{l} / \mathrm{s}$ ion getter pump. The vacuum system is baked out at $400^{\circ} \mathrm{C}$ for about 60 hours on a mercury diffusion-pump system with two liquid-nitrogen traps at a pressure of about $1 \times 10^{-6}$ torr. After closing the valve to the mercury diffusion pump and starting the ion getter pump, the pressure falls down to $1 \times 10^{-9}$ torr. For measuring the influence of ion bombardment the ion getter pump was replaced by a turbomolecular pump and the end pressure is $2 \times 10^{-9}$ torr. The total pressure is measured with a Bayard-Alpert ionisation gauge; in some measurements the partial pressure is measured with a quadrupole mass spectrometer.

The voltage is supplied by a transformer with rectifier giving an output of max. $90 \mathrm{kV}$ d.c. with a ripple smaller than $1 \%$. The prebreakdown current is measured either with a Philips $\mu$-voltmeter $\left(10^{-10}-10^{-2} \mathrm{~A}\right)$ or with a Keithly electrometer $601\left(10^{-10}-10^{-6} \mathrm{~A}\right)$. 
4. Experimental results. 4.1. Conditioning by sparks and ion bombardment. The basic pressure of the vacuum system is $1 \times 10^{-9}$ torr. In order to obtain this pressure and to maintain stable prebreakdown currents, it is necessary to bake out the system. Then the bake-out currents are measured, which are in very good agreement with the Fowler-Nordheim relation (1). Only the first series of measurements give sometimes a plot, deviating from a straight line. It seems that during the first voltage rise, a slight conditioning of the cathode occurs (see fig. 3). The Fowler-Nordheim plot was generally measured from $10^{-10} \mathrm{~A}$ to $10^{-6} \mathrm{~A} ; \beta$ and $A$ were calculated from this plot, assuming a value for the work function of stainless steel $4.7 \mathrm{eV}$ and of copper $4.5 \mathrm{eV}$.

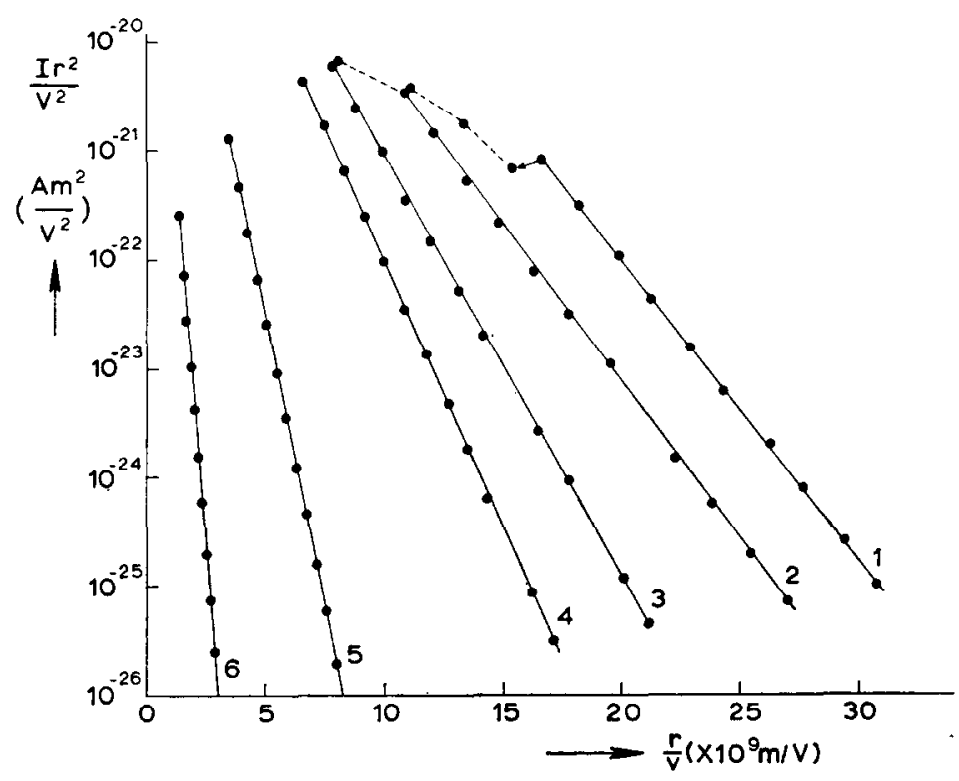

Fig. 3. Some Fowler-Nordheim plots for stainless steel. $1: \beta=101 ; A=5.7 \times 10^{-17} \mathrm{~m}^{2}$, first measurement. $2: \beta=101 ; A=3.8 \times 10^{-18} \mathrm{~m}^{2}$, second measurement. $3: \beta=67 ; A=7.2$ $\times 10^{-17} \mathrm{~m}^{2}$, third measurement. $4: \beta=53 ; A=1.2 \times 10^{-16} \mathrm{~m}^{2}$ after 1 spark. $5: \beta=27$; $A=1.1 \times 10^{-16} \mathrm{~m}^{2}$ aftcr 6 sparks. $6: \beta=10 ; A=4.5 \times 10^{-15} \mathrm{~m}^{2}$ after 20 sparks.

Metal electrodes in vacuum can be conditioned by sparks, because protrusions on the surface of the cathode are then destroyed by the field-emission current and by bombardment with anode material. However, the latter procedure can create new protrusions, resulting in a lower limit for $\beta$. Furthermore it is shown by Alpert $e t$ al. that high electric fields can cause protrusions ${ }^{2}$ ).

If the geometry of the electrodes is such that an anode-initiated breakdown is unlikely, we expected $\beta$ to decrease more than for plane-parallel electrodes. For the electrode configuration of fig. 2 the field $E$ at the cathode surface is $\beta V / r$, 
where $r$ is the radius of the tip ( $=0.10 \mathrm{~mm}$ for stainless steel and 0.15 for copper); it is not expected that a breakdown will start at the anode.

To investigate the conditioning by sparks the current-voltage characteristic was measured from $10^{-10} \mathrm{~A}$ upwards until breakdown occurs. This was repeated until the breakdown voltage no longer increased. If the breakdown voltage remains constant during at least 10 sparks the electrodes are assumed to be fully conditioned. Because the field-enhancement factor $\beta$ is a rather good indication for the condition of a vacuum gap, $\beta$ was measured as a function of the number of breakdowns and plotted in fig. 4. For stainless steel $\beta$ decreases very rapidly to about 10 and after prolonged sparking to about 8 . For copper the lowest value measured for $\beta$ was about 30 . Thus, for this electrode configuration and for stainless steel and copper cathodes $\beta$ is a factor 5 and 2, respectively, lower than for plane-parallel electrodes ${ }^{13}$ ).

To investigate the effect of ion bombardment on the emitting properties of the cathode, a Fowler-Nordheim plot was taken before the bombardment at a pressure of $2 \times 10^{-9}$ torr. Then argon was introduced in the vacuum system at a pressure of $1 \times 10^{-3}$ torr. This pressure was maintained by pumping with a turbomolecular pump. The voltage $V$ necessary for maintaining a constant prebreakdown current $I_{0}$ was measured as a function of time. It appeared that $V$ increases towards a new value $V_{\mathrm{m}}$. In fig. $5 \mathrm{~V} / V_{0}$ is plotted as a function of time. From this figure it appears that $V_{\mathrm{m}} / V_{0}$ is larger if $I_{0}$ is larger.

If the current did not change any more, the voltage was reduced to zero, the argon was pumped away with the turbomolecular pump, and a Fowler-Nordheim plot was measured at a pressure of $2 \times 10^{-9}$ torr. Some values of $\beta$ and $A$ before $\left(\beta_{1}\right.$ and $\left.A_{1}\right)$ and after conditioning $\left(\beta_{2}\right.$ and $\left.A_{2}\right)$ by ion bombardment of a copper cathode for the plots of fig. 5 are mentioned in table I.

TABLE I

\begin{tabular}{ccccc}
\hline \multicolumn{5}{r}{ Field-enhancement factors and emitting areas before and after ion bombardment } \\
\hline $\begin{array}{c}I_{0} \\
\text { (A) }\end{array}$ & $\beta_{1}$ & $\beta_{2}$ & $\begin{array}{c}A_{1} \\
\left(\mathrm{~m}^{2}\right)\end{array}$ & $\begin{array}{c}A_{2} \\
\left(\mathrm{~m}^{2}\right)\end{array}$ \\
\hline $1 \times 10^{9}$ & 54.5 & 53.5 & $2.5 \times 10^{16}$ & $1.7 \times 10^{-17}$ \\
$1 \times 10^{-8}$ & 54.5 & 20.0 & $2.5 \times 10^{-16}$ & $1.5 \times 10^{-15}$ \\
$1 \times 10^{-6}$ & 59.2 & 17.6 & $3.1 \times 10^{-17}$ & $9.4 \times 10^{-16}$ \\
$1 \times 10^{-5}$ & 54.5 & 24.6 & $2.5 \times 10^{-16}$ & $3.5 \times 10^{18}$ \\
\hline
\end{tabular}

After ion bombardment the field-enhancement factor is always lower, but the value of the emitting area after bombardment is unpredictable. For the stainlesssteel cathode the phenomena are about the same as for copper. The only difference is that $A_{2}$ is always larger than $A_{1}$ and the minimum value for $\beta$ is 7 . 


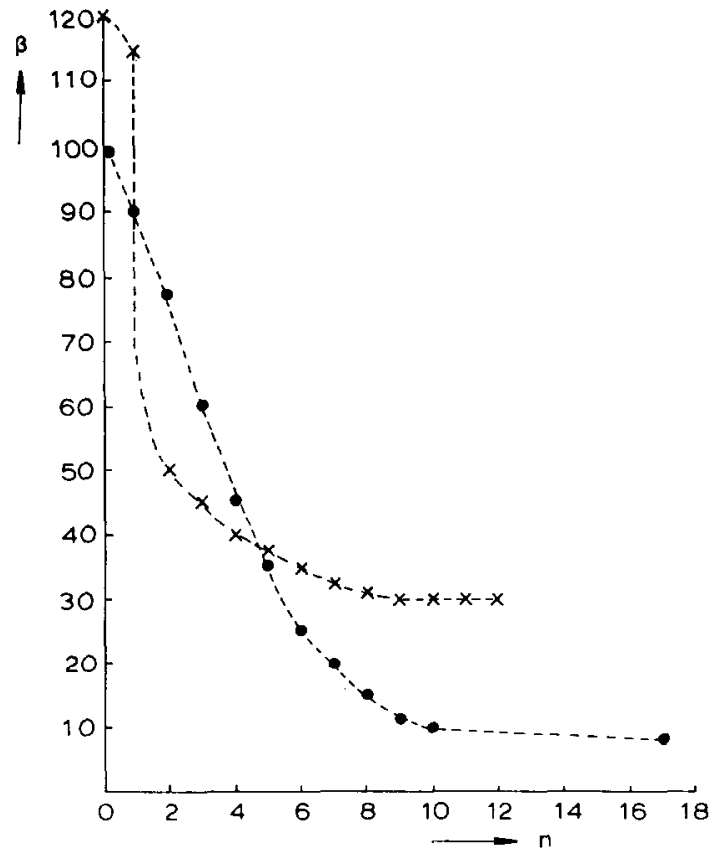

Fig. 4. $\beta$ as a function of the number of breakdowns: - stainless steel cathode; $\times$ copper cathode.

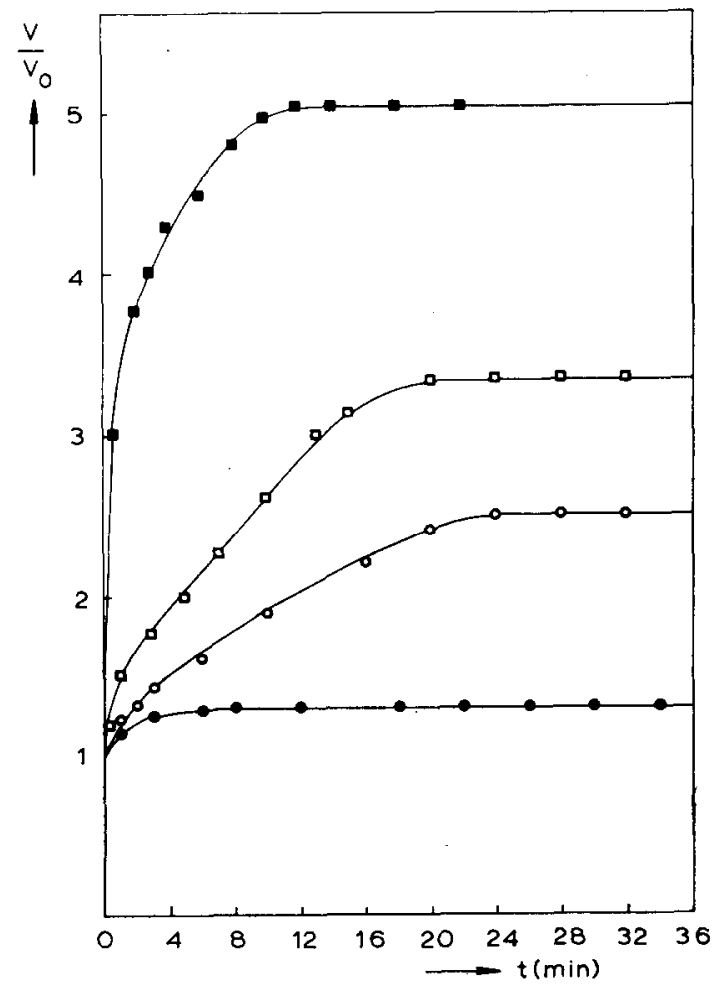

Fig. 5. Variation of $V / V_{0}$ for a copper cathode during ion bombardment as a function of time for different currents $I_{0}: \bullet I_{0}=1 \times 10^{-9} \mathrm{~A} ; \bigcirc I_{0}=1 \times 10^{-8} \mathrm{~A} ; \square I_{0}=1 \times 10^{-6} \mathrm{~A}$; $I_{0}=1 \times 10^{-5} \mathrm{~A}$. 
The ions bombarding the protrusion are most likely produced in the neighbourhood of the emitting area, where the field is strongly inhomogeneous. The distance over which the field is strongly divergent is about $r_{\mathrm{c}}{ }^{17}$ ), whiere $r_{\mathrm{c}}$ is the radius of the emitting area. So the maximum ion-sputtering voltage is of the order $V_{\mathrm{s}}=(\beta V / r) r_{\mathrm{c}}$, which for the measurements of table $\mathrm{l}$ is between 70 and $10 \mathrm{~V}$. For stainless steel $V_{\mathrm{s}}$ is between 200 and $20 \mathrm{~V}$. This may be an argument that the field-enhancement factor after sputtering is lower for stainless steel than for copper.

It is evident from fig. 5 and table I that the ratio $V_{\mathrm{m}} / V_{0}$ is not a measure for the ratio $\beta_{2} / \beta_{1}$, because the alteration in the emitting area is not predictable.

Probably, emitters with a very small emitting area $\left(A<1 \times 10^{-17} \mathrm{~m}^{2}\right)$ are unstable. For instance, in the example mentioned in the last line of table $I$, the Fowler-Nordheim plot was measured for currents up to $10^{-7} \mathrm{~A}$. The same plot was measured again 24 hours later. Up to a current of $10^{-7} \mathrm{~A}$ both plots were the same, indicating that a pressure of $2 \times 10^{-9}$ torr does not change the emitting properties of the cathode for a period of 24 hours. However, if one increases the current to $3 \times 10^{-7} \mathrm{~A}$ only a very small voltage increase is necessary. The FowlerNordheim plot measured afterwards gave a straight line up to $10^{-6} \mathrm{~A} ; \beta$ and $A$ calculated from this plot were 18.5 and $4.2 \times 10^{-16} \mathrm{~m}^{2}$, respectively. So, without any breakdown, $\beta$ was reduced by a factor $\frac{4}{3}$ and $A$ increased by a factor 120 .

In conclusion, ion bombardment of the cathode reduces $\beta$ considerably. Because the alteration in the emitting area is not predictable, a Fowler-Nordheim plot must be measured to get information about the emitting properties of the cathode. A high ratio of $V_{\mathrm{m}}$ to $V_{0}$ is not always a proof for a very well-conditioned electrode system.

4.2. Deconditioning after ion bombardment at high electric fields without breakdown. In the previous section it has been shown that ion bombardment causes a better condition of the cathode surface than sparking, assuming that $\beta$ is a measure for the conditioning: the lower the $\beta$, the better the conditioning of the electrodes.

As mentioned in section 4.1 it appears that if the emitting area is very small (between $10^{-18}$ and $10^{-17} \mathrm{~m}^{2}$ ), there is a transition to another state (with lower $\beta$ and much higher $A)$ at low currents $\left(10^{-7} \mathrm{~A}\right)$ without any indication of a breakdown. One cannot say this transition is a deconditioning, because a lower $\beta$ and a larger $A$ is an advantage from the point of view of the breakdown induced by field-emission electrons. But it seems that after ion bombardment the breakdown mechanism is altered and can better be described by the Cranberg mechanism ${ }^{13}$ ).

Also in the case of a reproducible Fowler-Nordheim plot (up to $10^{-6} \mathrm{~A}$ ) it is not possible to maintain currents larger than $10^{-7} \mathrm{~A}$ for a long time at a constant voltage. Both for the copper and the stainless-steel cathode it is necessary to decrease the voltage continuously to maintain a constant current at a value above $10^{-7} \mathrm{~A}$. 
The values of $\beta$ and $A$ before and after deconditioning ( $\beta_{2}$ resp. $\beta_{3}, A_{2}$ resp. $\left.A_{3}\right)$ for some measurements are mentioned in table II. The time to reach $\beta_{3}$ is a function of $I_{0}$ and is about $10 \mathrm{~min}$ for a current of $3 \times 10^{-4} \mathrm{~A}$ and about $50 \mathrm{~min}$ for a current of $10^{-6} \mathrm{~A}$.

TABLE II

Field-enhancement factors and emitting areas before and after deconditioning for different currents

\begin{tabular}{llcccc}
\hline Material & $\begin{array}{c}I_{0} \\
(\mathrm{~A})\end{array}$ & $\beta_{2}$ & $\beta_{3}$ & $\begin{array}{c}A_{2} \\
\left(\mathrm{~m}^{2}\right)\end{array}$ & $\begin{array}{c}A_{3} \\
\left(\mathrm{~m}^{2}\right)\end{array}$ \\
\hline Copper & $1 \times 10^{-7}$ & 20.5 & 20.6 & $1.5 \times 10^{-15}$ & $1.1 \times 10^{-15}$ \\
Copper & $5 \times 10^{-5}$ & 17.5 & 60.4 & $9.4 \times 10^{-15}$ & $1.1 \times 10^{-17}$ \\
Copper & $1 \times 10^{-4}$ & 19.0 & 42.0 & $6.3 \times 10^{-16}$ & $1.9 \times 10^{-17}$ \\
Copper & $3 \times 10^{-4}$ & 20.6 & 52.0 & $1.1 \times 10^{-15}$ & $1.8 \times 10^{-16}$ \\
Stainless steel & $1 \times 10^{-6}$ & 11.8 & 23.5 & $4.3 \times 10^{-16}$ & $3.2 \times 10^{-17}$ \\
Stainless steel & $1 \times 10^{-6}$ & 7.5 & 46.6 & $7.1 \times 10^{-15}$ & $3.0 \times 10^{-18}$ \\
\hline Stainless steel & 1 break- & & & & \\
& down & 46.6 & 9.0 & $3.0 \times 10^{-18}$ & $1.7 \times 10^{-14}$ \\
\hline
\end{tabular}

a $\beta$ and $A$ before and after breakdown, if the cathode is deconditioned by the electric field.

In the last line of table II the values of $\beta$ and $A$ before and after breakdown are given if the cathode is deconditioned by the electric field.

Summarizing, bombardment of the cathode by Ar ions decreases the fieldenhancement factor efficiently, resulting in an increased breakdown voltage. After one breakdown the condition of the vacuum gap is the same as after spark conditioning. At high electric fields there is a deconditioning effect after ion bombardment which is not found after spark conditioning. After deconditioning by the field, $\beta$ increased by more than a factor 3 and sometimes even a factor 6 . So, conditioning of a vacuum gap by ion bombardment is only useful if one breakdown is allowed and the pre-breakdown current does not rise above $10^{-7} \mathrm{~A}$ for a long time (i.e. one hour).

4.3. Breakdown in ultra high vacuum. For the configuration of the electrodes of fig. 2 only cathode-initiated breakdowns can be expected. After the experiments no traces were found on the anode surface, as in the case of planeparallel clectrodes. The measurements indicated indecd, that the breakdown mechanism is cathode-initiated; the maximum prebreakdown currents $I_{\mathrm{b}}$ are between the values $I_{\mathrm{c}}$ (cyl) and $I_{\mathrm{c}}$ (cone) ( $c f$. section 2.2). The maximum power just before breakdown is far above $P_{\mathrm{cc}}$. Hence, one must conclude that the Nottingham heating effect as given in the formulae (13) and (14) is not correct. The transition temperature $T_{\mathrm{c}}$ must be lower than that given by (10). Both for copper and stainless steel $T_{\mathrm{c}}$ is well below the melting temperature and Nottingham cooling occurs at breakdown. 


\section{TABLE III}

Breakdown parameters for copper determined from the combined measurements of prebreakdown characteristics and breakdown voltage

\begin{tabular}{cccccccc}
\hline$\beta$ & $\begin{array}{c}A \\
\left(\mathrm{~m}^{2}\right)\end{array}$ & $\begin{array}{c}I_{\mathrm{c}}(\mathrm{cyl}) \\
(\mathrm{mA})\end{array}$ & $\begin{array}{c}I_{\mathrm{c}}(\mathrm{cone}) \\
(\mathrm{mA})\end{array}$ & $\begin{array}{c}P_{\mathrm{cc}}(\mathrm{cyl}) \\
(\mathrm{W})\end{array}$ & $\begin{array}{c}P_{\mathrm{cc}}(\mathrm{cone}) \\
(\mathrm{W})\end{array}$ & $\begin{array}{c}I_{\mathrm{b}} \\
(\mathrm{mA})\end{array}$ & $\begin{array}{r}P_{\mathrm{exp}} \\
(\mathrm{W})\end{array}$ \\
\hline 42.5 & $1.5 \times 10^{-17}$ & 0.38 & 2.59 & 0.15 & 0.59 & 0.5 & 14 \\
54.0 & $1.0 \times 10^{-16}$ & 0.76 & 6.68 & 0.25 & 1.19 & 0.6 & 16 \\
29.9 & $1.1 \times 10^{-16}$ & 1.48 & 7.01 & 0.84 & 2.26 & 3.0 & 118 \\
30.8 & $2.8 \times 10^{-16}$ & 2.28 & 11.19 & 1.26 & 3.50 & 2.5 & 88 \\
\hline
\end{tabular}

TABLE IV

\begin{tabular}{rcccccccc}
\hline \multicolumn{10}{c}{ As table III, but now for stainless steel } \\
\hline \multicolumn{10}{c}{\begin{tabular}{c}
\multicolumn{1}{c}{$A$} \\
$\left(\mathrm{~m}^{2}\right)$
\end{tabular}} & $\begin{array}{c}I_{\mathrm{c}}(\mathrm{cyl}) \\
(\mathrm{mA})\end{array}$ & $\begin{array}{c}I_{\mathrm{c}}(\mathrm{cone}) \\
(\mathrm{mA})\end{array}$ & $\begin{array}{c}P_{\mathrm{cc}}(\mathrm{cyl}) \\
(\mathrm{W})\end{array}$ & $P_{\mathrm{cc}}(\mathrm{cone})$ & $\begin{array}{c}I_{\mathrm{b}} \\
(\mathrm{W})\end{array}$ & $\begin{array}{c}P_{\mathrm{exp}} \\
(\mathrm{mA})\end{array}$ & $(\mathrm{W})$ \\
\hline 10.1 & $3.0 \times 10^{-17}$ & 0.08 & 0.12 & 0.14 & 0.13 & 0.12 & 10 \\
9.4 & $2.6 \times 10^{-16}$ & 0.27 & 0.34 & 0.48 & 0.41 & 0.35 & 25 \\
46.6 & $3.0 \times 10^{-18}$ & 0.004 & 0.032 & 0.002 & 0.009 & 0.03 & 0.8 \\
12.1 & $1.2 \times 10^{-16}$ & 0.13 & 0.23 & 0.20 & 0.21 & 0.22 & 16.4 \\
\hline
\end{tabular}

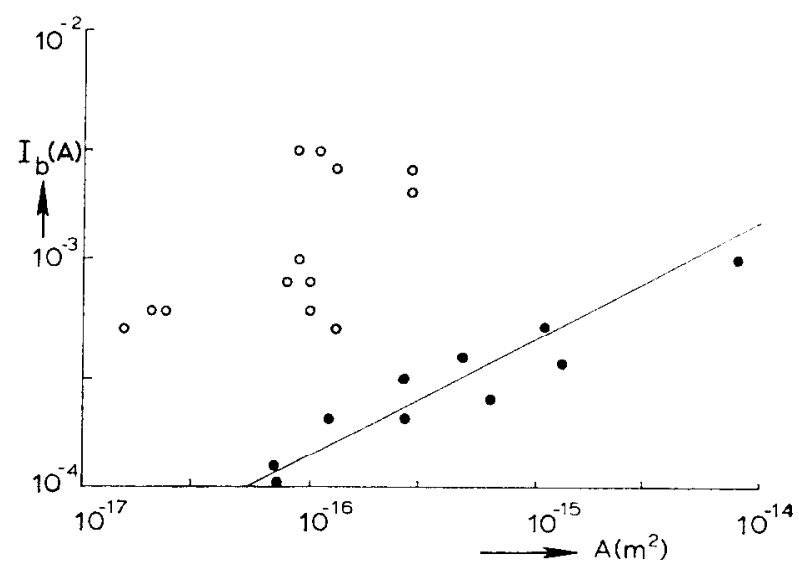

Fig. 6. Maximum prebreakdown current $I_{\mathrm{b}}$ for different emitting areas: - stainless steel; $O$ copper.

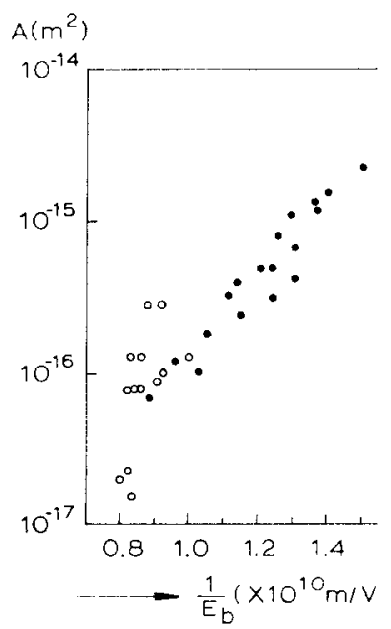

Fig. 7. Variation of $1 / E_{\mathrm{b}}$ wit emitting area $A: \bullet$ stainless steel; $O$ copper. 
Some measurements and matched values of the critical breakdown parameters are given in table III (for copper) and IV (for stainless steel).

From the measurements it is clear, that for the copper cathode the maximum prebreakdown current $I_{\mathrm{b}}$ lies between $I_{\mathrm{c}}$ (cyl) and $I_{\mathrm{c}}$ (cone); it is suggested that the emitting protrusions have shapes like a truncated cone with half angles between 0 and $10^{\circ}$. The protrusions on a stainless-steel cathode can be described as truncated cones with a half angle of about $10^{\circ}$. This is confirmed by fig. 6 and fig. 7. In fig. $6 I_{\mathrm{b}}$ is plotted as a function of the emitting area. It is expected from (8) and (9) that if the protrusions have always the same shape, $\log I_{b}$ versus $\log A$ is a straight line with slope $\frac{1}{2}$. If the shapes vary the spreading of the points is large. The plot $\log I_{\mathrm{b}} v s . \log A$ for stainless steel is in rather good agreement with (9), while the points for copper show a large deviation. In fig. $7 \log A$ is plotted as a function of $1 / E_{\mathrm{b}}$. For cylinders $E_{\mathrm{b}}$ must be almost constant for different areas (if Nottingham heating is neglected), except for large variations in the height of the protrusion. If the protrusions are truncated cones with constant half angle, $E_{\mathrm{b}}$ is a function of the emitting area. Because we do not know the exact shape of the emitting protrusions we can only indicate that the variation of $E_{\mathrm{b}}$ with $A$, as expected for truncated cones, is in agreement with fig. 7.

5. Discussion. 5.1. Temperature and space charge effects. The current-voltage characteristics plotted as $\log \left(I r^{2} / V^{2}\right) v s .(r / V)$ are mostly straight lines from $10^{-10} \mathrm{~A}$ upwards until breakdown occurs. Only above $10^{-4} \mathrm{~A}$ there is sometimes a slight deviation from the Fowler-Nordheim equation in such a way, that the current rises less than predicted by eq. (7). The Fowler-Nordheim equation (1) was derived for zero temperature. At finite temperature there are electrons above the Fermi level and these are more likely to penetrate the potential barrier. If ${ }^{8}$ )

$$
E>8.83 \times 10^{5} \phi^{\frac{1}{2}} T
$$

eq. (1) may be extended to:

$$
j_{T}=j_{0} \frac{\pi k T / d}{\sin (\pi k T / d)},
$$

where $j_{0}$ is the current density of eq. (1) at zero temperature, $k$ is the Boltzmann constant and $d$ can be approximated by $q$ of eq. (11), divided by the electron charge $e$.

In fig. $8 E=8.83 \times 10^{5} \phi^{\frac{1}{2}} T$ is plotted for $\phi=4.6 \mathrm{eV}$. Above this line eq. (16) holds for the current density $j_{T}$ as a function of $E, \phi$ and $T$. Because measurable field emission starts at a field of about $3 \times 10^{9} \mathrm{~V} / \mathrm{m}$ it is seen that for copper and stainless steel eq. (16) may be applied up to breakdown. 
For the derivation of the eqs. (8) and (9) it was assumed that, if the temperature of the emitting protrusion reaches a value of $800 \mathrm{~K}$ by its own field emission current, an instability occurs. At this temperature and at a field of $7 \times 10^{9} \mathrm{~V} / \mathrm{m}$ the current density is $j_{T} \approx 1.1 j_{0}$. At this high electric field the current density reaches a value of about $10^{12} \mathrm{~A} / \mathrm{m}^{2}$ and space-charge effects cannot be neglected. They lower the current density in comparison with eq. (1). Temperature and space charge at high electric fields have opposite effects and it is not possible to draw conclusions from a Fowler-Nordheim plot about the temperature of a protrusion at the onset of breakdown.

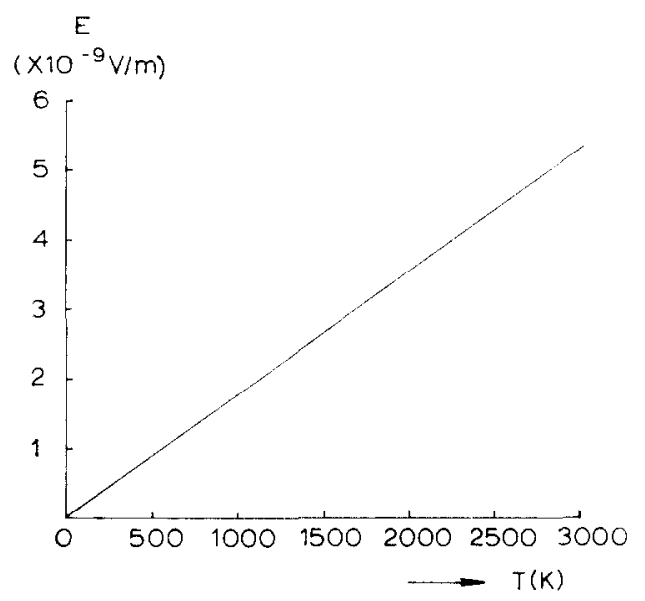

Fig. 8. $E=8.83 \times 10^{5} \phi^{\frac{1}{2}} T$ for $\phi=4.6 \mathrm{eV}$. Above this line eq. (16) holds for the current density $j_{T}$ as a function of $E, \phi$ and $T$.

5.2. Effect of the value of the work function on the breakdown mechanism. As mentioned in section 4.1, the work function $\phi$ of the cathode material is taken $4.5 \mathrm{eV}$ for copper and $4.7 \mathrm{eV}$ for stainless steel.

The influence of variations in the work function on the calculated emitting area can be neglected if $4<\phi<7 \mathrm{eV}$.

The field-enhancement factor $\beta$ varies with $\phi^{3 / 2}$ and so $I_{\mathrm{c}}$ (cyl) with $\phi^{-3 / 2}$. $I_{\mathrm{c}}$ (cone) does not vary with $\phi$. Because the exact shape of the protrusions is not known, we cannot derive the real value of $\phi$ from the measurements.

The influence of the variation of $\phi$ on the transition temperature $T_{\mathrm{c}}$ [eq. (10)] is much more important. From eq. (10) it follows that

$$
T_{\mathrm{c}}=5.32 \times 10^{-7} \frac{\beta V}{r} \phi^{-\frac{1}{2}} \approx \phi .
$$

In the tables V and VI $E$ and $T_{\mathrm{c}}$ are given for some values of $\phi$. 
TAble V

\begin{tabular}{cccc}
\hline $\begin{array}{c}\text { Electric field } \\
\text { perature as functions of the work function } \phi \text { for copper }\end{array}$ \\
& $\begin{array}{c}E \\
(\mathrm{~V} / \mathrm{m})\end{array}$ & $\begin{array}{c}T_{\mathrm{c}} \\
(\mathrm{K})\end{array}$ & $\begin{array}{c}T_{\mathrm{m}} \\
(\mathrm{K})\end{array}$ \\
\hline 4.5 & $1.0 \times 10^{10}$ & 2500 & 1356 \\
4.3 & $9.4 \times 10^{9}$ & 2390 & 1356 \\
4.0 & $8.4 \times 10^{9}$ & 2224 & 1356 \\
2.4 & $3.9 \times 10^{9}$ & 1332 & 1356 \\
\hline
\end{tabular}

Table V shows that only for an unrealistically low value of the work function $T_{\mathrm{c}}<T_{\mathrm{m}}$ and we must conclude that the theoretical value $T_{\mathrm{c}}$ is not correct. A work function of $4.0 \mathrm{eV}$ for stainless steel might be possible. However, it is not clear why the free-electron model should hold for stainless steel and not for copper.

TABLE VI

\begin{tabular}{|c|c|c|c|}
\hline$\phi$ & $\begin{array}{c}E \\
(\mathrm{~V} / \mathrm{m})\end{array}$ & $\begin{array}{c}T_{\mathrm{c}} \\
(\mathrm{K})\end{array}$ & $\begin{array}{r}T_{\mathrm{m}} \\
(\mathrm{K})\end{array}$ \\
\hline 4.7 & $8.0 \times 10^{9}$ & 1963 & 1723 \\
\hline 4.5 & $7.5 \times 10^{9}$ & 1880 & 1723 \\
\hline 4.3 & $7.0 \times 10^{9}$ & 1796 & 1723 \\
\hline 4.0 & $6.3 \times 10^{9}$ & 1670 & 1723 \\
\hline
\end{tabular}

\section{Conclusions}

1. By a configuration of the electrodes in which the anode does not play a role in the breakdown mechanism, the field-enhancement factor $\beta$ can be reduced by sparking to about 10 for stainless steel and 30 for copper.

2. Ion bombardment of the cathode surface reduces $\beta$ to 7 and 20 , respectively, if the ions are formed by field-emission electrons.

3. At high electric fields, particularly if the current is $>10^{-7} \mathrm{~A}$, there is a deconditioning.

4. Ion bombardment is only useful if not more than one breakdown is allowed and no currents $>10^{-7} \mathrm{~A}$ are admitted for a long time.

5. The breakdown mechanism can be considered as cathode-initiated.

6. It seems that the transition temperature at which Nottingham heating passes into Nottingham cooling is lower than the temperature predicted by the theory based on the free-electron model. 
Acknowledgement. The author is indebted to Professor G. A.W. Rutgers and to H.J. van Ark for many helpful discussions and suggestions during this work.

\section{REFERENCES}

1) Charbonnier, F. M., Bennette, C.J. and Swanson, L.W., J. appl. Phys. 38 (1967) 627.

2) Tomaschke, H.E. and Alpert, D., J. vacuum Sci. and Technol. 4 (1967) 192.

3) Cornish, J. C.L., Thesis, West Ham College of Techn., London (1970).

4) Llewellyn Jones, F. and Owen, W.D., Proc. Phys. Soc. 83 (1964) 283.

5) Alpert, D., Lee, D., Lyman, E. M. and Tomaschke, H.E., J. appl. Phys. 38 (1967) 880.

6) Janssen, A.P. and Jones, J.P., J. Phys. D 4 (1971) 118.

7) Fowler, R.H. and Nordheim, L., Proc. Roy. Soc. A119 (1928) 173.

8) Good, R.J. and Müller, E.W., Handbuch der Physik, Springer Verlag (Berlin, 1956), Vol. 21 p. 176.

9) Utsumi, T., Thesis, Cornell University (1967).

10) Jüttner, B., Rohrbeck, W. and Wolff, H., Beitr. Plasmaphys. 10 (1970) 383.

11) Dolan, W.W., Phys. Rev. 91 (1953) 510.

12) Chatterton, P. A., Proc. Phys. Soc. 88 (1966) 231.

13) Beukema, G.P., to be published.

14) Vibrans, G., J. appl. Phys. 35 (1964) 2855.

15) Charbonnier, F.M., Strayer, R.W., Swanson, L.W. and Martin, E.E., Phys. Rev. Letters 13 (1964) 397.

16) Swanson, L.W., Crouser, L.C. and Charbonnier, F. M., Phys. Rev. 151 (1966) 327.

17) Lewis, T. J., I. appl. Phys. 26 (1955) 1405. 